\title{
Microembolism after Endovascular Treatment of Unruptured Cerebral Aneurysms: Reduction of its Incidence by Microcatheter Lumen Aspiration
}

\author{
Dae Yoon Kim, MD', , Jung Cheol Park, MD², Jae Kyun Kim, MD³, Yu Sub Sung, PhD $^{4}$, \\ Eun Suk Park, MD', 5 , Jae Hyuk Kwak, MD', , \\ Choong-Gon Choi, MD, PhD', Deok Hee Lee, MD, PhD'
}

Purpose: Diffusion-weighted MR images (DWI) obtained after endovascular treatment of cerebral aneurysms frequently show multiple high-signal intensity (HSI) dots. The purpose of this study was to see whether we could reduce their incidence after embolization of unruptured cerebral aneurysms by modification of our coiling technique, which involves the deliberate aspiration of the microcatheter lumen right after delivery of each detachable coil into the aneurysm sac.

Materials and Methods: From January 2011 to June 2011, all 71 patients with unruptured cerebral aneurysms were treated using various endovascular methods. During the earlier period, 37 patients were treated using our conventional embolization technique (conventional period). Then 34 patients were treated with a modified coiling technique (modified period). DWI was obtained on the following day. We compared the occurrence of any DWI HSI lesions and the presence of the symptomatic lesions during the two time periods.

Results: The incidence of the DWI HSI lesions differed significantly at 89.2\% (33/37) during the conventional period and $26.5 \%(9 / 34)$ during the modified period $(p<0.0001)$. The incidence of symptomatic lesions differed between the two periods ( $29.7 \%$ during the conventional period vs. $2.9 \%$ during the modified period, $\mathrm{p}<0.003$ ).

'Department of Radiology, Research Institute of Radiology, Asan Medical Center, University of Ulsan College of Medicine, Seoul, Korea ${ }^{2}$ Department of Neurosurgery, Asan Medical Center, University of Ulsan College of Medicine, Seoul, Korea

${ }^{3}$ Department of Radiology, Chung-Ang University College of Medicine, Seoul, Korea

${ }^{4}$ Biomedical Imaging infrastructure, Department of Radiology, Research Institute of Radiology, Asan Medical Center, University of Ulsan College of Medicine, Seoul, Korea

${ }^{5}$ Department of Neurosurgery, Ulsan University Hospital, University of Ulsan College of Medicine, Ulsan, Korea

${ }^{6}$ Department of Neurology, Daegu Fatima Hospital, Daegu, Korea

${ }^{7}$ Department of Radiology, Bundang Jesaeng Hospital, Gyeonggi-do, Korea

Received March 26, 2015;

accepted after revision May 20, 2015.

Correspondence to: Deok Hee Lee, MD, PhD, Department of Radiology, Asan Medical Center, 88, Olympic-ro 43-gil, Songpa-gu, Seoul 138736, Korea.

Tel. 82.2.3010.5944 Fax. 82.2.476.0090 E-mail: dhlee@amc.seoul.kr

This is an Open Access article distributed under the terms of the Creative Commons Attribution Non-Commercial License (http://creativecommons.org/licenses/by-nc/3.0) which permits unrestricted non-commercial use, distribution, and reproduction in any medium, provided the original work is properly cited. 
Conclusion: Aspiration of the inner content of the microcatheter right after detachable coil delivery was helpful for the reduction of the incidence of microembolisms after endovascular coil embolization for the treatment of unruptured cerebral aneurysms.

Key Words : Intracranial Aneurysms; Coil embolization; Microembolism; Diffusion-weighted image

It is not unusual to observe tiny, dot-like, high-signalintensity (HSI) lesions on DWI after conventional cerebral angiography [1]. These lesions have been suspected to be 'microembolic infarcts' probably related to tiny thrombi, fragmented atherosclerotic plaque or air bubbles liberalized or introduced during catheter insertion and/or injection of contrast media or flushing saline. The incidence of microembolic infarcts has been reported to be even much greater after endovascular aneurysm treatment [2-12].

We were not free from those microembolic phenomena, although most of those lesions were asymptomatic or transiently symptomatic. We tried to reduce the occurrence by minimizing possible thromboembolisms. At first, our attention was drawn to the antithrombotic regimen $[13,14]$. Therefore, we began to give dual antiplatelets (aspirin and clopidogrel) more than five days before the procedure, regardless of stent use. Even the triple regimen which included cilostazol was given to patients who showed poor biochemical clopidogrel response, although it was not successful [15].

While searching for possible causes of the microembolisms, we found that a significant amount of air bubbles might have been introduced by various devices, including the various detachable embolization coils which were currently available in the market [16].

Considering this fact, we hypothesized that we could decrease the occurrence of microembolic lesions by reducing air-bubble introduction through a modification of our embolization procedure. Since we observed that most of the air bubbles were seen right after removal of the coil pusher in our previous report [16], we thought that air embolism from the coiling procedure could be minimized if we stopped flushing the microcatheter while regurging back the inner content of the microcatheter after detachment of the coils. Theoretically, since discontinuation of the pressurized saline flushing during embolization could result in clogging of the microcatheter, we decided to limit the discontinuation duration to be as short as possible.

The purpose of this study was to see whether we could reduce the incidence of micro-embolisms after embolization of unruptured cerebral aneurysms by procedural modification. We compared the incidence of the DWI HSI dots before and after application of our modified technique.

\section{MATERIALS AND METHODS}

\section{Study design and patients}

This retrospective study was approved by the institutional review board of our hospital. Individual patient's consent for the analysis of the data was waived. Since the primary follow-up imaging modality in monitoring aneurysm recurrence after coiling is time-of-flight (TOF) MR angiography (MRA) at our institution, we routinely obtain TOF MRA the following day after embolization of both ruptured and unruptured aneurysms. After observation of not infrequent occurrence of either symptomatic or asymptomatic microembolic lesions despite of our various efforts to reduce their occurrence, we started to include DWI sequence in our baseline MRA protocol as part of our daily practice from January 2011.

Since 'microcatheter lumen aspiration' was our latest activity to reduce the microembolic lesions, and the technical modification started to be applied in our practice from April 2011, we designated the patients who were treated in the prior 3 months as the control group (conventional group) and those treated in the post 3 months as the case group (modification group).

During the 6 months of the study period, we had a total of 72 consecutive patients who underwent endovascular treatment of unruptured cerebral aneurysms. Among them, we could not obtain an MR image in one patient who had a cardiac pacemaker. This patient was not symptomatic after the procedure and was excluded from the analysis.

No other procedural condition differed between the two groups except for the modification of microcatheter manipulation (microcatheter lumen aspiration) in the modification group. There were 37 patients in the conventional group and 34 in the modification group. 


\section{Microembolism Reduction after Endovascular Treatment}

\section{Embolization procedure during the conventional period}

All patients were on a dual antiplatelets regimen (Aspirin $75 \mathrm{mg}$ qd, clopidogrel $75 \mathrm{mg}$ qd) for at least five days, preferably for 10 days, before the procedure and without a loading dose. P2Y12 inhibition assay (VerifyNow P2Y12 Assay; Accumetrics, CA, USA) was performed on admission. When the test results showed a PRU value of 240 or higher, we loaded 200 mg of cilostazol (Pletaal; Otsuka Pharmaceutical Co., Japan) followed by $50 \mathrm{mg}$ twice a day in addition to the dual antiplatelets, regardless of stent use.

After obtaining informed consent for the coiling procedure, each patient was placed under general anesthesia. Systemic heparinization was achieved with a target activated clotting time (ACT) level between 200-250 seconds after placing a vascular access sheath, followed by a 5- or 6-Fr guiding catheter (Envoy; Codman Neurovascular or Chaperon; Microvention) placement. $1000 \mathrm{U} / \mathrm{h}$ of heparin was then given.

After placement of a microcatheter, embolization coils were inserted into the aneurysm sac. A variety of different coils, including Trufill Orbit coils (Codman, Raynham, MA), Axium coils (Covidien, Mansfield, MA), Hydrosoft coils (Microvention, Tustin, CA), and Target coils (Stryker, Fremont, CA) were used to fill the sac. Although coils were selected at the operator's discretion, various coils were used in combination in treating an aneurysm. A variety of different assisted methods (multiple-microcatheter technique, balloonassisted technique, stent-assisted technique, and combined technique) were applied. The balloonassisted technique was performed using Hyperform or Hyperglide balloons (Covidien). Two types of stents, a Neuroform stent (Stryker) and an Enterprise stent (Codman) were used based on anatomy or the individual operator's preference. After completion of the procedure, the patients were transferred to the neurosurgical intensive care unit (NSICU) without reversal of the systemic heparinization.

\section{Modified procedure during the modification period}

The detailed procedure was basically the same as was applied in the conventional group. The only difference was deliberate aspiration of the microcatheter lumen right after removal of each detached coil pusher, as some of us already were doing right after microcatheter navigation into the aneurysm sac, and after removal of the microguidewire to clear up the inner content of the microcatheter in the beginning of the procedure.

Since the air bubbles were noted mostly after removal of the coil pusher in our previous experiement [16], we decided to stop pressured saline flushing, right before removal of the coil pusher, until the inner content was aspirated out. To minimize the potential risk of microcatheter clogging we tried to shorten the discontinuation period as much as possible.

The following is the detail of the modified technique. After completion of detachment of each coil, the 3-way valve connected to the pressurized flushing system was closed, and then the coil pusher was removed. After disconnection of the rotating hemostatic valve from the microcatheter hub, inner content of the microcatheter was aspirated back gently with minimal application of negative pressure using a 2-mL syringe until a small amount of fresh blood was filling the inner space of the hub, since the potential space of the microcatheter lumen was only about $0.3 \mathrm{~mL}$. The blood in the hub was cleared up with saline using a jobby before being connected to the hemostatic valve to be flushed by pressurized saline again. The aspiration procedure was deliberately and repeatedly applied to the finishing coil. Two experienced operators shared the same modified technique. Occurrence of any microcatheter obstruction was carefully monitored.

\section{Post-procedural care and medication}

In uneventful cases, patients were observed for one day in the NSICU and for another day in the general ward before their discharge. They were on dual (or triple) antiplatelets before discharge. For simple coiling cases which did not require any stent, the patients were given a four-week course of aspirin mono-therapy (100 mg daily). If a stent was implanted or if there was any coil loop herniation into the parent artery, we placed the patient on a two-month course of dual antiplatelets followed by aspirin monotherapy for four months or more. No patient was put on triple-antiplatelet regimen after discharge.

\section{MR imaging and analysis}

As the baseline for the follow-up MR angiography, we routinely obtained TOF-MRA and DWI on postprocedural day 1 . Most of the images were obtained approximately 24 hours following the procedure.

DWI was obtained using a 3.0T Achieva MR imaging system (Philips Medical Systems, Best, the Netherlands). The single-shot, spin-echo, echo-planar imaging technique was used with a $b$ value of 1000 $\mathrm{s} / \mathrm{mm}^{2}$ and had the following imaging parameters: echo time, $56 \mathrm{~ms}$; repetition time, $3000 \mathrm{~ms}$; field of view, $250 \mathrm{~mm} \times 250 \mathrm{~mm}$; matrix size, $128 \times 128$; slice 
thickness, $5 \mathrm{~mm}$ with a gap of $2 \mathrm{~mm}$; number of slices, 20; number of average, 1. Parallel imaging was not applied. The ADC map was generated automatically from the acquisition console.

DWI images were reviewed by a neuroradiologist (CGC), who had more than 10 years of clinical experience in stroke imaging, using a PACS system. This reader classified HSI dots which could not be explained as artifacts, normal cortical signal or white matter bundles as infarction. Although there were no size criteria for the HSI dots, we regarded a lesion larger than $10 \mathrm{~mm}$ in maximum diameter as an overt infarct lesion. All HSI dots, including overt infarct lesions, were counted by the reader.

To enhance the reliability of the reader's interpretation, another reader (DYK) was asked to perform a semi-automatic interpretation process with a semiautomatic method using the in-house software. [See the appendix to know the semi-automatic method in detail.] Another neuroradiologist (DYK) with two years of stroke-imaging clinical experience classified whether the lesions detected by the in-house software were microembolic infarctions or not. Finally, the number of those lesions was automatically counted by the in-house software. If there was any discrepancy between the first reader's interpretation results and the software-detected results, consensus was reached between the two readers.
On clinical follow up, the occurrence of any neurological change was considered as symptomatic, whether or not the symptom or sign was transient.

The values are presented as the median and interquartile range (IQR). The Fisher's exact test and the Mann Whitney U-test were used for statistical analysis (SPSS ver. 11; Chicago, IL, USA). A two-sided $\mathrm{P}$ value $<0.05$ was considered significant. For analysis of the microembolic lesions on post-procedural DWI, we compared the DWI-positive (the presence of any DWI HSI dot) rates between the two time groups. We also compared the incidence of symptomatic microembolic lesions. The total number of DWI HSI dots was compared between the two time groups.

\section{RESULTS}

There was no significant difference in the basic characteristics between the two groups (Table 1). There was no significant procedural episode, such as microcatheter clogging, intraprocedural platelet aggregation, distal thromboembolism, or intraprocedural aneurysm rupture in either group.

Variable numbers of DWI HSI lesions were noted in $89.2 \%(33 / 37)$ in the conventional group and $26.5 \%$ $(9 / 34)$ in the modification group, a statistically significant difference $(p<0.0001)$. Other parameters were also favorable in the modification group (Table 2).

Table 1. Basic Demographic Data, Angiographic, and Procedural Data of the Patients in the Two Period Groups

\begin{tabular}{|c|c|c|c|c|}
\hline & $\begin{array}{l}\text { Total } \\
(n=71)\end{array}$ & $\begin{array}{l}\text { Conventional Period } \\
\qquad(n=37)\end{array}$ & $\begin{array}{l}\text { Modified Period } \\
\qquad(\mathrm{n}=34)\end{array}$ & $\mathrm{p}$ value \\
\hline Sex: Female (\%) & $60(84.5)$ & $31(83.8)$ & $29(85.3)$ & 1.000 \\
\hline Age: median (IQR) & $57(52-57)$ & $56(52-56)$ & $60(52-60)$ & 0.408 \\
\hline Location: Posterior circulation (\%) & $14(19.7)$ & $5(13.5)$ & $9(26.5)$ & 0.170 \\
\hline Diabetes (\%) & $6(8.5)$ & $3(8.1)$ & $3(8.8)$ & 0.914 \\
\hline Hypertension (\%) & $39(54.9)$ & $22(59.5)$ & $17(54.9)$ & 0.424 \\
\hline PRU: median (IQR) & $263(209-263)$ & $272(210-272)$ & $256(199-256)$ & 0.391 \\
\hline Percent inhibition: median (IQR) & $25(17-25)$ & $25(15-25)$ & $25(17-25)$ & 0.898 \\
\hline Max. diameter of the index aneurysm: median (IQR) & $4.7(3.7-5.2)$ & $4.7(3.8-4.7)$ & $5.2(3.7-5.2)$ & 0.917 \\
\hline Multiple aneurysm embolization & $9(12.7)$ & $5(13.5)$ & $4(11.8)$ & 1.000 \\
\hline Double microcatheter technique (\%) & $13(18.3)$ & $10(27.0)$ & $3(8.8)$ & 0.066 \\
\hline Stent-assisted technique (\%) & $28(39.4)$ & $18(48.6)$ & $10(29.4)$ & 0.098 \\
\hline Balloon-assisted technique & 0 & 0 & 0 & - \\
\hline Procedural duration in minutes: median (IQR) & $63(43-63)$ & $73(49-73)$ & $51(35-51)$ & 0.725 \\
\hline
\end{tabular}

Note: IQR = inter-quartile range 


\section{Microembolism Reduction after Endovascular Treatment}

\section{DISCUSSION}

Even though we could not completely avoid microembolic phenomena during the embolization of unruptured intracranial aneurysms, we could reduce its incidence by modification of our microcatheter manipulation technique based on our prior observation [16].

In previous reports, the incidence of DWI abnormality varied after endovascular treatment of both ruptured and unruptured aneurysms (Table 3) [2-12]. Although those rates were obtained under variable conditions, our conventional group's result was the worst. However, we were able to reduce the rate to those of lower-rate groups after modification of the technique.
The exact pathomechanism of DWI HSI lesions (microembolism) is not well understood, and it is beyond the scope of our current study. Some of the earlier reports regarded the lesions as resulting from thromboembolisms [2, 11, 17]. However, thromboembolisms alone could not explain the mechanism, and there had been no witnessed intra- or post-procedural acute platelet aggregation or thromboembolic phenomenon in our patients, even in the conventional group. Brooks et al. reported that they experienced intraprocedural thrombosis requiring treatment in $10(6.5 \%)$ of 155 cases [4]. Interestingly, they could not observe a DWI abnormality in six of their 10 overt thrombosis cases [4].

Furthermore, in the conventional group, microembolic lesions were continually seen, even with strict

Table 2. Incidence of the DWI High-signal Intensities between the Two Period Groups

\begin{tabular}{|c|c|c|c|c|}
\hline & $\begin{array}{l}\text { Total } \\
(\mathrm{n}=71)\end{array}$ & $\begin{array}{l}\text { Conventional Period } \\
\qquad(\mathrm{n}=37)\end{array}$ & $\begin{array}{l}\text { Modified Period } \\
\qquad(\mathrm{n}=34)\end{array}$ & $p$ value \\
\hline Number of patients with DWI positivity (\%) & $42(59.2)$ & $33(89.2)$ & $9(26.5)$ & $<0.0001$ \\
\hline Number of DWI HSI dots: median (IQR) & $4(0-4)$ & $33(8-52)$ & $0(0-0)^{*}$ & $<0.0001$ \\
\hline Number of symptomatic patients (\%) & $12(16.9)$ & $11(29.7)$ & $1(2.9)$ & 0.003 \\
\hline
\end{tabular}

Note: $\mathrm{HSI}=$ high-signal intensity, IQR = inter-quartile range, * Since the number of HSI in this period was too small, median and IQR were all 0 .

Table 3. Incidence of DWI Abnormality after Endovascular Treatment of Both Ruptured and Unruptured Aneurysms in the Literature

\begin{tabular}{|c|c|c|c|c|c|c|}
\hline Authors & Year & Aneurysm status & $\begin{array}{l}\text { Patient } \\
\text { number }\end{array}$ & $\begin{array}{l}\mathrm{DWI}(+) \\
\text { rate }(\%)\end{array}$ & $\begin{array}{l}\text { Symptomatic } \\
\text { rate }(\%)\end{array}$ & Note \\
\hline Biondi et al. ${ }^{3}$ & 2000 & Ruptured and unruptured & 20 & 10 & 0 & \\
\hline Rordorf et al. $^{9}$ & 2001 & Unruptured & 14 & 57 & 7.1 & \\
\hline Soeda et al. ${ }^{11}$ & 2003 & Unruptured & 66 & 61 & 40 & Balloon-assisted in $49 \%$ \\
\hline Albayram et al. ${ }^{2}$ & 2004 & Ruptured and unruptured & 20 & 20 & 10 & Balloon-assisted technique only \\
\hline 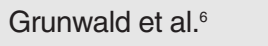 & 2006 & Unruptured & 50 & 42 & 2 & \\
\hline Brooks et al. ${ }^{4}$ & 2008 & Ruptured and unruptured & 132 & 24 & 7.5 & $\begin{array}{l}\text { Only including treated vascular } \\
\text { territory lesions }\end{array}$ \\
\hline Chung et al. ${ }^{5}$ & 2008 & Ruptured and unruptured & 163 & 60.1 & 6 & \\
\hline Spiotta et al. ${ }^{12}$ & 2011 & Unruptured & 147 & 21.5 & 3.8 & Balloon-assisted in 81 patients \\
\hline Sim et al..$^{10}$ & 2012 & Ruptured and unruptured & 39 & 43.6 & 10.3 & \\
\hline Hahnemann et al. ${ }^{7}$ & 2013 & Unruptured & 75 & 64 & 6.7 & Stent-assisted coiling cases only \\
\hline Kang et al. ${ }^{8}$ & 2013 & Unruptured & 343 & 54.5 & 4.1 & Data from two medical institutions \\
\hline $\begin{array}{l}\text { Current study } \\
\text { conventional period }\end{array}$ & & Unruptured & 37 & 89.2 & 29.7 & \\
\hline $\begin{array}{l}\text { Current study } \\
\text { modified period }\end{array}$ & & Unruptured & 34 & 26.5 & 2.9 & \\
\hline
\end{tabular}




\section{Dae Yoon Kim, et al.}

antiplatelet pretreatment in addition to systemic heparinization. We added cilostazol when biochemical resistance of clopidogrel was suggested by a VerifyNow assay. To our disappointment, this was not helpful in any way.

Among the various coils on the market, the amount of air bubble was the greatest from the Target Detachable Coil system (Stryker). We could not identify a mechanism for the significant air bubble generation from that particular coil at that time. The subsequent experiment performed by the company could unveil that it was the air trapped in the lumen of the coil pusher, which was made of a hypotube. Now, the coil does not generate that many air bubbles after modification of the hypotube purging method by creating a ventilation hole at the proximal end of the coil pusher [not published].

Then, the main cause of the microembolisms was the air bubbles. Since the purpose that we started to aspirate the microcatheter was exactly to prevent potential air embolisms, we had no choice but to conclude that it was due to air bubbles. However, we would like to reserve this conclusion until we can prove the direct cause-and-effect relationship between the two. Actually, we cannot exclude other possible sources, such as fragmented hydrophilic coating materials, which were liberated during the procedure due to friction between the devices. There have been several reports on this issue since the first introduction of hydrophilic coating technology for neurointervention [18-21]. Further research is needed to prove the exact pathomechanism of the microembolism, since the problem is not solely limited to aneurysm coiling procedures. The reported incidence of microembolisms is even greater after flow diversion procedures. What is important to us as of now is that, regardless of the nature of the embolic material, we could prevent its introduction into the cerebral circulation by simple modification of our embolization technique.

As we mentioned in the introduction, there is a theoretical concern regarding the safety of repeated microcatheter aspiration. Many of us rely heavily on the importance of continuous flushing of the microcatheter from the beginning of the procedure to prevent any obstruction or thromboembolic phenomena from the microcatheter. In that situation, one may have a significant concern about the safety of intentional discontinuation of saline flushing, and even aspiration of the microcatheter lumen. On the other hand, since interventionists in our group were already accustomed to the aspiration procedure, which we always did in the microcatheter manipulation during placement of the tip into the aneurysm sac, we did not experience any difficulty in the application of the technique for the detachable coil insertion. There was no case of intentional microcatheter removal due to failure of aspiration or clogging of the microcatheter. The only caveat for this technical modification was the need of repeated 'disconnection-aspiration-reconnection' steps, which could be bothersome or look tedious.

Regarding the interpretation of DWI HSI dots, previous reports simply analyzed the presence of DWIpositivity, and one or two interpreters counted the number of DWI HSI dots with or without a systematic consensus process [1-12]. In some reports, the diameter of the lesions or the volume of the DWI lesion load was measured. However, we found that sometimes lesion detection is not easy, and for this reason we used the 'semi-automatic method' in order to reduce potential human bias. First of all, the software was able to detect HSI lesions sensitively and reproducibly with a constant threshold. With this, we thought that the reliability or interobserver agreement in lesion detection would be increased by minimization of human interpretation error. In addition, the possibility to miss the true lesion is likely to be lowered due to decreased human perception error.

There are several limitations to this study. In the study design, we simply divided the two time periods as part of our clinical practice and not as an experimental, study-based cohort design. Therefore, we were not free from a potential limitation of retrospective analysis with a rather small number of patients. We were also not able to completely deny any unnoticed change in our procedural pattern between the two time periods.

We believe that every endovascular procedure harbors the same problem of microembolic phenomena, and the risk could be higher in the future as the procedures and devices become more complex. We should pay more attention to get rid of this potentially hazardous phenomenon, from the development of new devices to the application to our patients.

In conclusion, we could reduce the incidence of postprocedural microembolisms on DWI after coil embolization of unruptured cerebral aneurysms by modification of the procedure, which involves the deliberate aspiration of the inner content of the microcatheter after removal of each coil pusher.

\section{Appendix}

The in-house software was based on the AFNI, a free software, consisting of a set of $\mathrm{C}$ programs for mapping 


\section{Microembolism Reduction after Endovascular Treatment}

human brain activity and developed by the Medical College of Wisconsin and the National Institutes of Health (AFNI, MCW and NIH, http://afni.nimh.nih. gov) and ImageJ, a public domain, Java-based, image processing program developed at the National Institutes of Health (NIH, Bethesda, MD, USA; http://rsbweb. nih.gov/ij/). At first, DWI was registered to a skullstripped brain atlas (TT_27, a Talairached version of the Colin_N27 dataset). After registration, we performed several processing steps in order to remove non-specific HSI changes, such as artifacts, normal cortical signal or white-matter bundles. The in-house software with high sensitivity detected any DWI HSI lesions, which included these specific conditions: 1) size less than $10 \mathrm{~mm}$ in maximum diameter; 2) signal intensity above the certain threshold; and 3) circularity between $0.9 \sim 1.0$. Finally, the in-house software displayed the detection results of the DWI HSI dots to a second reader.

\section{Acknowledgement}

This article does not have any grants to declare.

The authors don't have any personal or institutional financial interest in drugs, materials, or devices described in this submission.

The authors gratefully acknowledge the technical support received from staff in the Biomedical Imaging Infrastructure, Department of Radiology, Asan Medical Center.

\section{References}

1. Bendszus M, Koltzenburg M, Burger R, Warmuth-Metz M, Hofmann E, Solymosi L. Silent embolism in diagnostic cerebral angiography and neurointerventional procedures: a prospective study. Lancet 1999;354:1594-1597

2. Albayram S, Selcuk H, Kara B, Bozdag E, Uzma O, Kocer N, et al. Thromboembolic events associated with balloon-assisted coil embolization: evaluation with diffusion-weighted MR imaging. AJNR Am J Neuroradiol 2004;25:1768-1777

3. Biondi A, Oppenheim C, Vivas E, Casasco A, Lalam T, Sourour $\mathrm{N}$, et al. Cerebral aneurysms treated by Guglielmi detachable coils: evaluation with diffusion-weighted MR imaging. AJNR Am J Neuroradiol 2000;21:957-963

4. Brooks NP, Turk AS, Niemann DB, Aagaard-Kienitz B, Pulfer K, Cook T. Frequency of thromboembolic events associated with endovascular aneurysm treatment: retrospective case series. $J$ Neurosurg 2008;108:1095-1100

5. Chung SW, Baik SK, Kim Y, Park J. Thromboembolic Events after Coil Embolization of Cerebral Aneurysms : Prospective Study with Diffusion-Weighted Magnetic Resonance Imaging Follow-up. J Korean Neurosurg Soc 2008;43:275-280

6. Grunwald IQ, Papanagiotou P, Politi M, Struffert T, Roth C, Reith
W. Endovascular treatment of unruptured intracranial aneurysms: occurrence of thromboembolic events. Neurosurgery 2006; 58:612-618; discussion 612-618

7. Hahnemann ML, Ringelstein A, Sandalcioglu IE, Goericke S, Moenninghoff C, Wanke I, et al. Silent embolism after stentassisted coiling of cerebral aneurysms: diffusion-weighted MRI study of 75 cases. J Neurointerv Surg 2014;6:461-465

8. Kang DH, Kim BM, Kim DJ, Suh SH, Kim DI, Kim YS, et al. MR-DWI-positive lesions and symptomatic ischemic complications after coiling of unruptured intracranial aneurysms. Stroke 2013;44:789-791

9. Rordorf G, Bellon RJ, Budzik RE, Farkas J, Reinking GF, Pergolizzi RS, et al. Silent thromboembolic events associated with the treatment of unruptured cerebral aneurysms by use of Guglielmi detachable coils: prospective study applying diffusionweighted imaging. AJNR Am J Neuroradiol 2001;22:5-10

10. Sim SY, Shin YS. Silent microembolism on diffusion-weighted MRI after coil embolization of cerebral aneurysms. Neurointervention 2012;7:77-84

11. Soeda A, Sakai N, Sakai H, Iihara K, Yamada N, Imakita S, et al. Thromboembolic events associated with Guglielmi detachable coil embolization of asymptomatic cerebral aneurysms: evaluation of 66 consecutive cases with use of diffusion-weighted MR imaging. AJNR Am J Neuroradiol 2003;24:127-132

12. Spiotta AM, Bhalla T, Hussain MS, Sivapatham T, Batra A, Hui $\mathrm{F}$, et al. An analysis of inflation times during balloon-assisted aneurysm coil embolization and ischemic complications. Stroke 2011; 42:1051-1055

13. Kang HS, Kwon BJ, Kim JE, Han MH. Preinterventional clopidogrel response variability for coil embolization of intracranial aneurysms: clinical implications. AJNR Am J Neuroradiol 2010; 31:1206-1210

14. Lee DH, Arat A, Morsi H, Shaltoni H, Harris JR, Mawad ME. Dual antiplatelet therapy monitoring for neurointerventional procedures using a point-of-care platelet function test: a singlecenter experience. AJNR Am J Neuroradiol 2008;29:1389-1394

15. Kono K, Shintani A, Yoshimura R, Okada H, Tanaka Y, Fujimoto $\mathrm{T}$, et al. Triple antiplatelet therapy with addition of cilostazol to aspirin and clopidogrel for Y-stent-assisted coil embolization of cerebral aneurysms. Acta Neurochir (Wien) 2013;155:1549-1557

16. Lee DH, Hwang SM, Lim OK, Kim JK. In vitro observation of air bubbles during delivery of various detachable aneurysm embolization coils. Korean J Radiol 2012;13:412-416

17. Derdeyn CP. Diffusion-weighted imaging as a surrogate marker for stroke as a complication of cerebrovascular procedures and devices. AJNR Am J Neuroradiol 2001;22:1234-1235

18. Barnwell SL, D’Agostino AN, Shapiro SL, Nesbit GM, Kellogg JX. Foreign bodies in small arteries after use of an infusion microcatheter. AJNR Am J Neuroradiol 1997;18:1886-1889

19. Fargen KM, Hoh BL. Ipsilateral cerebral hemorrhage following deployment of the Pipeline Embolization Device. J Neurosurg 2014;120:363-364

20. Mehta RI, Fishbein MC, Solis OE, Jahan R, Salamon N, Vinters $\mathrm{HV}$, et al. Intravascular polymer material after coil embolization of a giant cerebral aneurysm. Hum Pathol 2009;40:1803-1807

21. Mehta RI, Solis OE, Jahan R, Salamon N, Tobis JM, Yong WH, et al. Hydrophilic polymer emboli: an under-recognized iatrogenic cause of ischemia and infarct. Mod Pathol 2010;23:921-930 